\title{
Management of intractable pain in patients treated with hemorrhoidectomy for mixed hemorrhoids
}

\author{
Jingjuan Feng, Jian Cheng, Feng Xiang \\ Department of Anorectal Surgery, Sichuan Academy of Medical Sciences \& Sichuan Provincial People's Hospital, Chengdu, China \\ Contributions: (I) Conception and design: J Feng, F Xiang; (II) Administrative support: F Xiang; (III) Provision of study materials or patients: F Xiang, \\ J Cheng; (IV) Collection and assembly of data: J Feng, J Cheng; (V) Data analysis and interpretation: J Feng, F Xiang; (VI) Manuscript writing: All \\ authors; (VII) Final approval of manuscript: All authors. \\ Correspondence to: Feng Xiang. Department of Anorectal Surgery, Sichuan Academy of Medical Sciences \& Sichuan Provincial People's Hospital, \\ Chengdu, Sichuan, 610072, China. Address: No. 32, West Section 2, First Ring Road, Qingyang District, Chengdu 610072, China. \\ Email: xiangfeng1022@163.com.
}

\begin{abstract}
Background: Post-hemorrhoidectomy pain is common, usually temporary, and responsive to analgesics. However, some patients experience prolonged, intractable anal pain, which is refractory to conventional analgesics and adversely effects quality of life. We aimed to evaluate the efficacy of a combination injection containing local anesthesia and steroids for the treatment of intractable post-hemorrhoidectomy anal pain.

Methods: This study included five patients with intractable post-hemorrhoidectomy anal pain who presented between July 2015 and November 2018. Milligan-Morgan hemorrhoidectomy (M-M) with a sclerosant injection had been performed in three patients and stapled hemorrhoidectomy (SH) along with external hemorrhoidectomy in two. For the purpose of this study, all patients received local combination injections containing ropivacaine and triamcinolone acetonide. The primary outcome was the visual analogue scale (VAS) score, and secondary outcomes included the Pittsburgh Sleep Quality Index (PSQI) score, adverse reactions, and recurrence.

Results: Prior to treatment, the VAS score was 8 in one patient and 5-7 in four, and the PSQI score was $\geq 16$ in all patients. The injection was administered once $(n=4)$ or twice $(n=1)$. Rapid pain resolution was achieved on the day of treatment (VAS scores 0-3), and patients reported undisturbed sleep the same night. VAS scores were 0-2 (n=4) and 1-3 (n=1), and the PQSI scores were 0-6 (n=4) and 11-15 (n=1) at the 6-month follow-up. No patient reported significant pain or adverse reaction.
\end{abstract}

Conclusions: Intractable post-hemorrhoidectomy anal pain is rare but significantly effects quality of life. While conventional analgesia may be ineffective, the local injection of anti-inflammatory and analgesic drugs may be a useful treatment strategy.

Keywords: Hemorrhoids; hemorrhoidectomy; stapled hemorrhoidectomy (SH); intractable pain

Submitted Nov 11, 2020. Accepted for publication Jan 17, 2021.

doi: 10.21037/apm-20-2385

View this article at: http://dx.doi.org/10.21037/apm-20-2385

\section{Introduction}

Hemorrhoids are one of the most common anorectal disorders in clinical practice. Based on the Goligher clinical grading system (1), hemorrhoids are classified into grades I to IV. Grades I and II are usually manageable with conservative treatment, diet and lifestyle changes, application of topical ointments, ligation, or sclerosant injections, whereas Grades III and IV often require surgery. The most common surgical procedures used in the management of hemorrhoids are open, closed, or stapled hemorrhoidectomy ( $\mathrm{SH}$ ), and the MilliganMorgan hemorrhoidectomy (M-M) (2). Following hemorrhoidectomy, most patients experience different 
degrees of postoperative pain, which may cause anxiety (3).

Intractable post-hemorrhoidectomy anal pain is relatively rare, and its underlying pathophysiology remains unclear (4). Unfortunately, intractable pain is invariably recurrent, refractory to analgesics including morphine, and significantly affects the quality of life of patients (5). Locally injected drugs at the site of pain are widely used to control postoperative pain (6) because they are effective, simple to administer, rapid acting, and are associated with few and minor side effects (7). Although combination injections containing local anesthesia and steroids prolong the duration of analgesia (8) and administering these injections at the site of pain could provide long-lasting analgesic effects, to the best of our knowledge, there are no previous studies evaluating the efficacy of this treatment. Therefore, the present study aimed to evaluate the efficacy of a combination injection containing local anesthesia and steroids in patients with intractable perianal pain after hemorrhoidectomy. We present the following article in accordance with the MDAR checklist (available at http:// dx.doi.org/10.21037/apm-20-2385).

\section{Methods}

This study was approved by the Institutional Review Board of Sichuan Provincial People's Hospital and all patients provided written informed consent for their anonymized data to be used. The registration number is ChiCTR2000030738. All procedures performed in this study involving human participants were in accordance with the Declaration of Helsinki (as revised in 2013).

We retrospectively investigated five patients with intractable post-hemorrhoidectomy pain who were diagnosed at or referred to our hospital between July 2015 and November 2018. Three patients had undergone M-M along with sclerosant injection and two had undergone $\mathrm{SH}$ along with external hemorrhoidectomy. All patients presented with well-defined sites of pain which were easily palpated during digital rectal examination and recorded pain visual analogue scale (VAS) scores $\geq 6$ and Pittsburgh Sleep Quality Index (PSQI) scores $\geq 16$. All patients reported experiencing intractable pain that was intense enough to affect sleep and interfere with their daily lives and work activities and had been present for over 3 months. All patients received a local injection containing a combination of ropivacaine and triamcinolone at the site of pain. We analyzed the demographic data, surgical characteristics, and changes in pain and sleep at the time of visit, on the day of treatment, and at a 6-month follow-up. Analyses and comparison of the changes in VAS and PSQI range, and the number of adverse reactions and recurrence were conducted. GraphPad Prism 6.0 was used for statistics, unpaired Mann Whitney test and Wilcoxon matchedpairs signed rank test were used to estimate the VAS and PSQI scores before and after the treatment. $\mathrm{P}<0.05$ was considered significant.

\section{Results}

The clinical and demographic data of patients are shown in Table 1. Of the two men and three women included, four presented with pain at only one site and one presented with pain at two sites. All sites were located on the dentate line of the rectal mucosa, and no patient complained of diffuse or generalized pain. The two patients who had undergone $\mathrm{SH}$ experienced pain approximately $1.5 \mathrm{~cm}$ above the dentate line, close to the anastomosis and the three who had received sclerosant injection experienced pain approximately $1 \mathrm{~cm}$ above the dentate line at the site of the fibrotic scar.

All patients reported the use of various oral medications, topical analgesics, and sedatives prior to enrollment in the study as seen in Table 2. One reported the use of morphine to deal with pain and all patients consumed oral diazepam before going to bed. The PSQI score in all patients was $\geq 16$, indicating very poor sleep quality. The VAS scores of three patients were 6-7 indicating moderate pain and two had severe pain $(8,9)$. Four patients received a single injection, and only patient \#2 required two injections. Immediate pain relief was observed in all patients and VAS scores decreased by as much as 3 points within 30 minutes of the injection. At the 6-month follow up all patients reported no pain or occasional mild pain, the VAS scores were $0-3$ and PSQI scores were $0-10$. The maximal score after treatment was used in analysis. Both the VAS and PSQI scores were significant before and after treatment with unpaired Mann Whitney test, and close to be significant $(\mathrm{P}=0.0625)$ by Wilcoxon matched-pairs signed rank test. Considering the small sample size, these indicate a significant improvement in sleep quality (Table 3).

\section{Discussion}

Intractable pain after hemorrhoid surgery is relatively rare and the lack of effective interventions can severely affect daily activities (5). Prior to intervention, the patients in the present study had experienced intractable pain for over 
Table 1 Characteristics of patients with intractable pain

\begin{tabular}{lccccc}
\hline Patient & Age (years) & Sex & Course of disease (months) & Surgical features & Sites of pain (number) \\
\hline Patient 1 & 52 & M & 6 & M-M + sclerosant injection & 1 \\
Patient 2 & 67 & F & 3.5 & SH + external hemorrhoidectomy & M-M + sclerosant injection \\
Patient 3 & 63 & F & 5 & M-M + sclerosant injection & 1 \\
Patient 4 & 46 & M & 7 & SH + external hemorrhoidectomy & 1 \\
Patient 5 & 41 & F & 4 & 1 & \\
\hline
\end{tabular}

M-M, Milligan-Morgan hemorrhoidectomy; SH, stapled hemorrhoidectomy.

Table 2 Analgesics and sedatives used before the treatment

\begin{tabular}{|c|c|c|c|c|c|c|c|}
\hline Patient & \multicolumn{3}{|c|}{ Oral medications } & \multicolumn{4}{|c|}{ Topical medications } \\
\hline Patient 2 & - & + & - & + & + & - & + \\
\hline Patient 5 & + & + & - & + & + & - & + \\
\hline
\end{tabular}

+ indicates used, - indicates not used.

Table 3 Visual Analogue Scale (VAS) and Pittsburgh Sleep Quality Index (PSQI) scores before and after the treatment

\begin{tabular}{lcccc}
\hline Patient & $\begin{array}{c}\text { Mean VAS score before } \\
\text { the treatment }\end{array}$ & $\begin{array}{c}\text { VAS score at 6-month follow-up } \\
\text { after the treatment }\end{array}$ & $\begin{array}{c}\text { Mean PSQI score } \\
\text { before the treatment }\end{array}$ & $\begin{array}{c}\text { PSQI score at 6-month follow-up } \\
\text { after the treatment }\end{array}$ \\
\hline Patient 1 & 9 & $0-2$ & 20 & $0-10$ \\
Patient 2 & 6 & $1-3$ & 16 & $6-10$ \\
Patient 3 & 6 & $0-2$ & 17 & $0-5$ \\
Patient 4 & 7 & $0-2$ & 19 & $0-5$ \\
Patient 5 & 8 & $0-3$ & $0-10$ \\
\hline
\end{tabular}

$\mathrm{P}=0.0079$ by unpaired Mann Whitney test for both Mean PSQI score and Mean VAS score before and after the treatment; $\mathrm{P}=0.0625$ by Wilcoxon matched-pairs signed rank test for both Mean PSQI score and Mean VAS score before and after the treatment. VAS, visual analogue scale; PSQI, Pittsburgh Sleep Quality Index.

3 months, which significantly interfered with their ability to work and led to sleep disturbances. Marked psychological stress was reported by all patients and patient \#1 had remained in pain despite being admitted to a psychiatric hospital for 2 weeks. Pain had persisted or recurred in all patients despite oral medications and the administration of various topical analgesics and sedatives.

Few studies have described intractable pain following hemorrhoidectomy. Ortiz et al. (4) reported that only one among 27 patients who underwent $\mathrm{SH}$ showed unexplained, persistent pain for 3 months. However, Cheetham et al. (5) reported that at the follow-up of 16 patients who underwent $\mathrm{SH}$, five had experienced persistent pain and urgency for 15 months and two had severe pain and were unable to work. Although the exact pathophysiology remains unclear, it is unlikely that removal of a part of the rectal muscles contributes to this condition. No abnormalities in the appearance of the anus, the tone of the anal sphincter, or the 
lower segment of the rectum were seen in the present study and all patients presented with clearly identified sites of pain or trigger points that were easily palpated at the time of diagnosis. In many patients, minor nerve damage heals spontaneously during the usual postoperative period, and postoperative pain that persists beyond the time required for natural tissue healing is attributable to neuropathological causes (9). Thaha et al. (10) reported post-defecation pain syndrome in three patients who underwent $\mathrm{SH}$ and in whom oral nifedipine led to rapid pain relief. This pain was possibly rectal in origin and not associated with anal sphincter injury, as was previously assumed. SH may partially injure the submucosal plexus along with the underlying muscles and alter the neuroregulation of the rectal muscles leading to rectal hyperactivity and spasm. This may account for the pain felt by two patients who underwent $\mathrm{SH}$ in the present study. Local injection of antiinflammatory and analgesic drugs can alleviate hyperactivity and spasm and may have led to the relief in pain in these two patients, although the exact mechanism is uncertain.

Three patients in our study had received sclerotherapy. Sclerosant-induced local inflammation results in fibrosis, and the strong astringent action of sclerosants causes protein coagulation and vasoconstriction that promote the hardening and adhesion of hemorrhoidal tissue (11). Sclerosant injections for the treatment of hemorrhoids may cause serious complications such as rectourethral fistula (12), rectovaginal fistula (13), and perianal necrosis (14) as well as mild pain $(15,16)$ although few reports have described persistent pain (17).

Considering previous literature findings and the surgical treatment and pain characteristics observed in the patients in the present study, it is reasonable to conclude that the intractable pain observed in all patients in this study can be attributed to compression or stimulation of nerve endings by locally formed fibrotic scar tissue, which altered the neuroregulation of the anorectal muscles.

Local injections at the sites of pain are widely used as an effective treatment strategy for rapid pain relief in various conditions and local nerve blocks are highly effective for postsurgical analgesia (18). Several studies have recommended combination therapy with local anesthetics (for their rapid analgesic effects) and corticosteroids (for their slower but longer-acting action), and such combinations have often been used in clinical studies $(19,20)$. Therefore, we injected a combination of ropivacaine and triamcinolone locally at the site of pain to achieve longterm analgesic effect in patients with intractable post- hemorrhoidectomy pain. We observed rapid pain relief in all patients and no recurrence of pain was reported at the 6 months follow-up.

The retrospective design, small sample size, and singlecenter setting are limitations of our study. The actual pathophysiological mechanisms contributing to intractable post-hemorrhoidectomy pain, and the mechanism of action of ropivacaine and triamcinolone used to treat it require further investigation.

\section{Conclusions}

Local injection of ropivacaine and triamcinolone produced rapid and effective pain relief in patients with intractable post-hemorrhoidectomy pain. This treatment was associated with significantly decreased pain scores, significantly improved sleep quality, and no adverse reactions or recurrence. This treatment strategy may be beneficial for the treatment of intractable perianal pain in patients with similar clinical presentation.

\section{Acknowledgments}

Funding: None.

\section{Footnote}

Reporting Checklist: The authors have completed the MDAR checklist. Available at http://dx.doi.org/10.21037/apm-202385

Data Sharing Statement: Available at http://dx.doi. org/10.21037/apm-20-2385

Conflicts of Interest: All authors have completed the ICMJE uniform disclosure form (available at http://dx.doi. org/10.21037/apm-20-2385). The authors have no conflicts of interest to declare.

Ethical Statement: The authors are accountable for all aspects of the work in ensuring that questions related to the accuracy or integrity of any part of the work are appropriately investigated and resolved. This study was approved by the Institutional Review Board of Sichuan Provincial People's Hospital (No: 2020-4) and all patients provided written informed consent for their anonymized data to be used. All procedures performed in this study involving human participants were in accordance with the 
Declaration of Helsinki (as revised in 2013).

Open Access Statement: This is an Open Access article distributed in accordance with the Creative Commons Attribution-NonCommercial-NoDerivs 4.0 International License (CC BY-NC-ND 4.0), which permits the noncommercial replication and distribution of the article with the strict proviso that no changes or edits are made and the original work is properly cited (including links to both the formal publication through the relevant DOI and the license). See: https://creativecommons.org/licenses/by-nc-nd/4.0/.

\section{References}

1. Goligher J. Surgery of the Anus, Rectum and Colon. 5th edition. London: Bailliere Tindall, 1984.

2. Simillis C, Thoukididou SN, Slesser AA, et al. Systematic review and network meta-analysis comparing clinical outcomes and effectiveness of surgical treatments for haemorrhoids. Br J Surg 2015;102:1603-18.

3. Joshi GP, Neugebauer EA, PROSPECT Collaboration. Evidence-based management of pain after haemorrhoidectomy surgery. Br J Surg 2010;97:1155-68.

4. Ortiz H, Marzo J, Armendariz P. Randomized clinical trial of stapled haemorrhoidopexy versus conventional diathermy haemorrhoidectomy. Br J Surg 2002;89:1376-81.

5. Cheetham MJ, Mortensen NJM, Nyström P-O, et al. Persistent pain and faecal urgency after stapled haemorrhoidectomy. Lancet 2000;356:730-3.

6. Ventham NT, O'Neill S, Johns $\mathrm{N}$ et al. Evaluation of novel local anesthetic wound infiltration techniques for postoperative pain following colorectal resection surgery: a meta-analysis. Dis Colon Rectum 2014;57:237-50.

7. Bartley J, Han E, Gupta P, et al. Transvaginal trigger point injections improve pain scores in women with pelvic floor hypertonicity and pelvic pain conditions. Female Pelvic Med Reconstr Surg 2019;25:392-6.

8. Kim B, Lee H, Chung H, et al. The efficacy of topical bupivacaine and triamcinolone acetonide injection in the relief of pain after endoscopic submucosal dissection for gastric neoplasia: A randomized double-blind, placebocontrolled trial. Surg Endosc 2015;29:714-22.

9. Elahi F, Reddy C, Ho D. Ultrasound guided peripheral nerve stimulation implant for management of intractable pain after inguinal herniorrhaphy. Pain Physician. 2015;18:E31-8.

10. Thaha MA, Irvine LA, Steele RJ, et al. Postdefaecation pain syndrome after circular stapled anopexy is abolished by oral nifedipine. Br J Surg 2005;92:208-10.

11. Yuksel BC, Armagan H, Berkem H, et al. Conservative management of hemorrhoids: A comparison of venotonic flavonoid micronized purified flavonoid fraction (MPFF) and sclerotherapy. Surg Today 2008;38:123-9.

12. Tanwar R, Singh SK, Pawar DS. Rectourethral fistula: A rare complication of injection sclerotherapy. Urol Ann 2014;6:261-3.

13. Ray S, Mandal S, Khamrui S. Rectovaginal fistula: An extremely rare complication after injection sclerotherapy for hemorrhoids. Am Surg 2013;79:E143-4.

14. Elram R, Wasserberg N. Anorectal necrosis induced by injection sclerotherapy for hemorrhoids. Int J Colorectal Dis 2007;22:997-8.

15. Tokunaga Y, Sasaki H, Saito T. Evaluation of sclerotherapy with a new sclerosing agent and stapled hemorrhoidopexy for prolapsing internal hemorrhoids: retrospective comparison with hemorrhoidectomy. Dig Surg 2010;27:469-72.

16. Moser KH, Mosch C, Walgenbach M, et al. Efficacy and safety of sclerotherapy with polidocanol foam in comparison with fluid sclerosant in the treatment of firstgrade haemorrhoidal disease: A randomised, controlled, single-blind, multicentre trial. Int J Colorectal Dis 2013;28:1439-47.

17. Cocorullo G, Tutino R, Falco N, et al. The non-surgical management for hemorrhoidal disease. A systematic review. G Chir 2017;38:5-14.

18. Xu W, Varghese C, Bissett IP, et al. Network metaanalysis of local and regional analgesia following colorectal resection. Br J Surg 2020;107:e109-22.

19. Shah K, Watson D, Campbell C et al. Intra-articular injection composed of steroid, iohexol and local anaesthetic: Is it stable? Br J Radiol 2009;82:109-11.

20. Rudnik-Jansen I, Schrijver K, Woike N, et al. Intraarticular injection of triamcinolone acetonide releasing biomaterial microspheres inhibits pain and inflammation in an acute arthritis model. Drug Deliv 2019;26:226-36.

(English Language Editor: B. Draper)

Cite this article as: Feng J, Cheng J, Xiang F. Management of intractable pain in patients treated with hemorrhoidectomy for mixed hemorrhoids. Ann Palliat Med 2021;10(1):479-483. doi: 10.21037/apm-20-2385 